\title{
CIRURGIA DO CONTORNO CORPORAL NO PACIENTE APÓS PERDA PONDERAL MACIÇA: EXPERIÊNCIA DE TRÊS ANOS EM HOSPITAL PÚBLICO SECUNDÁRIO
}

\author{
Simone Cristina Orpheu ${ }^{1}$, Pedro Soler Coltro ${ }^{2 *}$, Gean Paulo Scopel ${ }^{1}$, Fábio Lopes Saito ${ }^{1}$, Marcus Castro Ferreira ${ }^{3}$ \\ Trabalho realizado na divisão de Cirurgia Plástica e Queimaduras do Hospital das Clínicas da Faculdade de Medicina da Universidade de São Paulo, S.Paulo, SP
}

\author{
${ }^{*}$ Correspondência: \\ Dr. Ovídio Pires de Campos, \\ no 171 - Apto. 618 - \\ Cerqueira César \\ São Paulo - SP \\ CEP: 05403-010 \\ Telefone: (11) 9190-5832 \\ Fax: (11) 3069-6636 \\ pscoltro@hotmail.com
}

\begin{abstract}
RESUMO
OвJEtıvo. Apresentar a experiência clínico-cirúrgica de três anos relacionada às cirurgias de contorno corporal no paciente após perda ponderal maciça, realizadas pela equipe de Cirurgia Plástica do Hospital Estadual de Sapopemba.

Métodos. Estudo retrospectivo realizado no Hospital Estadual de Sapopemba, vinculado ao Hospital das Clínicas da Faculdade de Medicina da Universidade de São Paulo, no período de julho de 2005 a julho de 2008. Foram operados 98 pacientes submetidos a tratamento de obesidade mórbida por meio de gastroplastia redutora, com sucesso para a técnica, ou emagrecimento por método não-cirúrgico. Os critérios de exclusão foram tabagismo, intenção gestacional e instabilidade ponderal, exigindo-se peso estável por pelo menos seis meses após atingido a perda ponderal satisfatória.

Resultados. Dos 98 pacientes operados, 97\% eram do sexo feminino, com idade média de 40,5 anos e o método de emagrecimento foi cirúrgico em 88\% deles. Das 177 cirurgias plásticas realizadas, $46 \%$ foram abdominoplastias, seguidas por mastoplastias (15\%), suspensões de coxa (13\%) e braquioplastias (12\%). O período médio de internação foi dois dias e as principais complicações diagnosticadas foram: seroma (28\%), necroses/deiscências de pequeno porte (18\%), necroses/ deiscências de grande porte (4\%), hematomas (4\%), tromboflebite superficial de veia safena (2\%) e trombose venosa profunda (1\%).

Conclusão. O desejo de cirurgias para melhora do contorno corporal após perda ponderal maciça constitui demanda crescente. Cabe ao cirurgião plástico conhecer as peculiaridades clínicas e os riscos maiores de complicações desses pacientes, ponderar suas expectativas, analisar os resultados e manter a busca constante pelo aperfeiçoamento das técnicas cirúrgicas.
\end{abstract}

Unitermos: Cirurgia Plástica. Obesidade mórbida.

\section{INTRODUÇÃO}

A obesidade constitui uma epidemia globalizada no mundo atual, especialmente nas nações ocidentais. Estimativas da Organização Mundial de Saúde (OMS) referem alarmantes 1,7 bilhões de pessoas em situação de sobrepeso ou obesidade ${ }^{1}$, atingindo $60 \%$ dos norte-americanos. A obesidade mórbida ${ }^{2,3}$ pode ser definida como um excesso ponderal de 100 libras (cerca de $45 \mathrm{Kg}$ ) acima do peso ideal, ou duas vezes o peso normal para altura ou ainda, como definido pelo Instituto Nacional de Saúde dos Estados Unidos, por um índice de massa corpórea (IMC) - relação entre o peso pelo quadrado da altura -superior a $35 \mathrm{Kg} / \mathrm{m}^{2}$ associado a comorbidades, ou acima de $40 \mathrm{Kg} / \mathrm{m}^{2}$ na ausência de comorbidades.

São comorbidades relacionadas à obesidade: diabetes não insulino-dependente, apnéia do sono, síndrome hipoventilatória torácica, hipertensão arterial, hiperlipidemia, doença coronariana, refluxo gastroesofágico, cálculos biliares, esteato-hepatite não-alcoólica, estados de hipercoagulação sanguínea (incluindo trombose venosa profunda e embolia pulmonar), incontinência urinária de estresse, infertilidade, amenorréia, enxaqueca, doença articular degenerativa, gota, depressão e ainda neoplasias de cólon, mama e útero, entre outras ${ }^{3,4}$. 0 impacto socioeconômico dessas comorbidades é elevado e nos EUA é superado somente pelos custos de tratamentos de doenças relacionadas ao tabagismo ${ }^{5}$.

O tratamento da obesidade inclui a prática de atividade física, o uso de substâncias anorexígenas, métodos comportamentais como os diários nutricionais, programas de acompanhamento psicológico e autoajuda. Entretanto, tais métodos confrontam inúmeras vezes com a teoria do "set point", pela qual o organismo

\footnotetext{
1. Médicos Assistentes da Disciplina de Cirurgia Plástica da Faculdade de Medicina da Universidade de São Paulo, São Paulo, SP

2. Médico Residente de Cirurgia Plástica do Hospital das Clínicas da Faculdade de Medicina da Universidade de São Paulo - USP, São Paulo, SP

3. Professor Titular da Disciplina de Cirurgia Plástica da Faculdade de Medicina da Universidade de São Paulo - USP, São Paulo, SP
} 
tende a defender um peso pré-determinado como método de biopreservação contra situações de inanição. Logo, o paciente pode emagrecer até seu peso ideal porém, metabolicamente, permanecerá obeso, implicando na recidiva da doença em médio prazo, findo o tratamento 4 .

A cirurgia bariátrica ${ }^{6,7}$ é o único tratamento atual efetivo no controle da obesidade mórbida. É indicada para pacientes que preencham os critérios de obesidade mórbida sem resposta aos programas não-cirúrgicos de emagrecimento. Desde a primeira publicação por Kremen, em 1954, os procedimentos cirúrgicos, mal-absortivos ou restritivos, evoluíram no tocante à via de acesso, que pode ser laparotômica convencional ou videolaparoscópica, e também em relação à redução nos índices de complicação e morbimortalidade. Esses avanços possibilitaram uma demanda populacional de obesos mórbidos crescente em busca da cirurgia. Atualmente, os procedimentos bariátricos mais realizados são a banda gástrica ajustável videolaparoscópica e o "bypass" em Y de Roux. Tais procedimentos implicam em perda ponderal esperada de no mínimo 50\% do excesso de peso, em um período de até 24 meses, em média, após a intervenção cirúrgica.

Após essa perda ponderal maciça, o paciente vivencia a nova condição de saúde, refletida pela melhora ou mesmo resolução das comorbidades, especialmente o diabetes tipo II, e pela própria perda de peso, com reflexos bio-psicossociais positivos. Entretanto, e quase simultaneamente, o paciente defronta-se com a nova imagem corporal. A perda do excesso de peso poderá produzir um corpo magro, normal ou ainda com sobrepeso, ou até mesmo obeso, a depender da gravidade da condição ponderal prévia. Não é infrequente que um paciente hiperobeso (IMC superior a $50 \mathrm{Kg} / \mathrm{m}^{2}$ ) apresente, após uma cirurgia bariátrica de sucesso, índices de obesidade.

Uma condição constante nessa nova imagem corporal é a flacidez cutânea associada à ptose das diversas regiões anatômicas, como mamas, braços, coxas, glúteos e tronco. Ao lado do impacto psicossocial do dermatocalázio generalizado, também há implicações médicas, com doenças como o intertrigo e limitações funcionais para deambulação, micção e atividade sexual ${ }^{8}$.

No escopo da cirurgia plástica, o tratamento do excesso de pele após perda ponderal maciça pode ser considerado desafiador, uma vez que atinge todo o corpo do paciente e requer estratégias para minimizar as complicações pelo cuidado integral, desde o planejamento das incisões até a avaliação dos resultados no período pós-operatório tardio.

A avaliação de resultados é complexa em cirurgia plástica, ao envolver parâmetros subjetivos e contar com poucas publicações comparativas, porém é necessária para permitir progressos. Em relação ao paciente após perda ponderal maciça, o resultado da cirurgia plástica, seja de dermolipectomias, ou pexias de tecidos ptóticos, o que se observa no período pós-operatório tardio é a manutenção de flacidez residual em graus variados a ponto de afligir o cirurgião, o paciente ou ambos.

Esses pacientes enfrentaram diversos programas de emagrecimento clínico antes de realizar a cirurgia bariátrica, portanto o revestimento cutâneo foi submetido a distensões e retrações seriadas, o chamado "efeito sanfona", o que pode comprometer a composição proteica responsável pela resistência e elasticidade da pele, especialmente o colágeno e a elastina ${ }^{9-16}$.

\section{ObJetivo}

Apresentar a experiência clínico-cirúrgica de três anos relacionada às cirurgias de contorno corporal no paciente após perda ponderal maciça, realizadas pela equipe de Cirurgia Plástica do Hospital Estadual de Sapopemba - SP.

\section{Métodos}

O projeto de pesquisa foi aprovado pela Comissão de Ética para Análise de Projetos de Pesquisa (Cappesq) do Hospital das Clínicas da Faculdade de Medicina da Universidade de São Paulo, ao qual o Hospital Estadual de Sapopemba é vinculado. A partir desta autorização, foram selecionados os participantes do projeto. Aos pacientes foi fornecido o termo de consentimento livre e esclarecido e a equipe médica colocou-se à disposição para dirimir eventuais dúvidas. Foi salientado que os pacientes poderiam desistir de sua participação no projeto a qualquer momento, sem qualquer implicação no tratamento dos mesmos. Também foram informados sobre a obtenção de fotografias pré, trans e pós-operatórias e solicitou-se autorização para o uso da imagem pessoal, quando justificada.

Os pacientes foram alocados do Hospital Estadual de Sapopemba e o período do estudo foi de julho de 2005 a julho de 2008. Foram operados 98 pacientes, submetidos a tratamento de obesidade mórbida por meio de gastroplastia redutora, com sucesso para a técnica, ou seja, perda ponderal de pelo menos $40 \%$ do excesso de peso, ou emagrecimento por método não cirúrgico. Os critérios de exclusão foram o tabagismo, a intenção gestacional e a instabilidade ponderal, exigindo-se peso estável por pelo menos 6 meses após ter sido atingida a perda ponderal satisfatória para cada caso.

A presença de comorbidades foi analisada detalhadamente na anamnese pré-operatória. A grande maioria dos pacientes, após o tratamento da obesidade, referia melhora ou completa resolução de diversas doenças sistêmicas. Entretanto, alguns ainda apresesentavam hipertensão arterial sistêmica (9\%), depressão em tratamento medicamentoso (8,5\%), hipotireoidismo $(6,5 \%)$, asma (3\%), diabete melito e dislipidemia ( $1 \%$ cada).

A rotina de preparo pré-operatório incluiu exames laboratoriais, de imagem e métodos gráficos em cardiologia, seguindo recomendações preconizadas pelo grau de "phisical status" das sociedades de anestesiologia vigentes no período.

A utilização de drenos de sucção foi rotina da equipe nas abdominoplastias e o dispositivo foi mantido por 7 a 14 dias, ou até débito inferior a $50 \mathrm{ml}$ por 24 horas. Por outro lado, seu uso nas demais áreas corporais tratadas não foi habitual, sendo a indicação individualizada para cada paciente.

A equipe cirúrgica foi composta por dois cirurgiões assistentes (o autor e um dos coautores, médicos especialistas em cirurgia plástica, regularmente contratados pelo hospital), por um médico residente de cirurgia plástica do Hospital das Clínicas da Faculdade de Medicina da USP, em periodicidade bimestral, e por uma instrumentadora cirúrgica. No primeiro 
ano desta casuística, o médico residente em questão cursava o $3^{\circ}$ ano de cirurgia plástica, e nos dois últimos anos da casuística o médico residente cursava $02^{\circ}$ ano de sua formação para especialista.

\section{Resultados}

Foram operados 98 pacientes após perda ponderal maciça, no período de julho de 2005 a julho de 2008. A distribuição por sexo foi 96 pacientes do sexo feminino e dois do sexo masculino. A idade variou de 17 a 64 anos, com média de 40,5 anos. 0 tempo de seguimento dos pacientes variou de 4 a 36 meses, com média de 20 meses.

Cerca de 50\% destes pacientes apresentavam escolaridade de nível fundamental, 35\% nível médio e 11\% nível superior. Quanto ao estado civil, 60\% dos pacientes eram casados e 30\% solteiros. 0 método de tratamento de obesidade foi cirúrgico em 86 pacientes e não-cirúrgico em 12 deles. A gastroplastia redutora foi laparotômica convencional em 74 pacientes e videolaparoscópica em 12 deles.

0 índice de massa corpórea (IMC) máximo durante a vida desses pacientes atingiu 78,42 kg/m² e o mínimo foi $38,41 \mathrm{~kg} /$ $\mathrm{m}^{2}$. Após o tratamento da obesidade, em fase de estabilidade ponderal, e anteriormente à realização da primeira cirurgia plástica de contorno corporal, esse grupo apresentava IMC variado (Gráfico 1): 27 pacientes com IMC acima de $30 \mathrm{~kg} / \mathrm{m}^{2}, 55 \mathrm{com}$ IMC entre 25 e $30 \mathrm{~kg} / \mathrm{m}^{2}$ e $16 \mathrm{com}$ IMC abaixo de $25 \mathrm{~kg} / \mathrm{m}^{2}$. O maior IMC anterior a cirurgia plástica de contorno corporal foi $36,98 \mathrm{~kg} / \mathrm{m}^{2}$.

O intervalo de tempo entre o tratamento da obesidade e a realização da primeira cirurgia de contorno corporal variou de 1,8 ano até 8 anos. Entretanto, o intervalo de tempo entre a primeira consulta do paciente com a equipe e a primeira cirurgia não superou seis meses.

No período estudado, foram realizadas 177 cirurgias, incluindo os procedimentos principais de contorno corporal e procedimentos menores como revisão de cicatrizes e cirurgias para tratamento de complicações. Dentre os 98 pacientes operados, 51 foram submetidos a uma cirurgia, 28 a duas cirurgias e 19 realizaram três ou mais cirurgias.

Em relação às regiões corporais tratadas, foram realizadas 81 abdominoplastias (Figuras 1 e 2), das quais 63 (77\%) foram abdominoplastias com componente vertical e horizontal ("em âncora") e 18 com incisão transversa inferior (22\%); 26 mastoplastias, das quais nove (34\%) com inclusão de implante de silicone gel texturizado; 23 dermolipectomias de região medial de coxas (Figura 1); 21 dermolipectomias de região medial dos braços; quatro dermolipectomias de tronco (superior, inferior ou torácica lateral); duas ritidoplastias e uma blefaroplastia isolada.

A permanência hospitalar dos pacientes variou de acordo com o tipo de cirurgia realizada. Nas abdominoplastias, os pacientes permaneceram internados por dois dias em 68\% casos, ou por três dias em 30\%. Apenas dois pacientes permaneceram internadas por período superior a sete dias pela necessidade de tratamento de complicação vascular requerendo anticoagulação. Nos procedimentos cirúrgicos para tratamento das demais regiões corporais, o tempo médio de internação foi de dois dias em $84 \%$ das intervenções realizadas.
A principal complicação sistêmica foi de origem vascular, representada por um evento de trombose venosa profunda (1\%), e dois eventos de tromboflebite superficial até croça de veia safena, requerendo anticoagulação (2\%). Não houve tromboembolismo pulmonar nesse grupo.

Dentre as complicações locais, destacam-se os seromas (28\%), hematomas (4\%), deiscências ou necroses de pequeno porte, com cicatrização espontânea em tempo inferior a 60 dias (18\%), deiscências ou necroses de grande porte, com cicatrização em até seis meses e com cirurgia complementar para revisão de cicatrizes (5\%).

Houve necessidade de reinternação para exploração cirúrgica em cinco pacientes, por hematoma de pequeno volume em coxa e abdome, e para drenagem de um abscesso de parede abdominal tardio (Dois meses após dermolipectomia abdominal "em âncora").

\section{Discussão}

Após a cirurgia bariátrica, o emagrecimento estimado é de aproximadamente $50 \%$ do excesso de peso, o que significa que inúmeros pacientes manterão índices de massa corpórea de sobrepeso ou obesidade ( 25 a $30 \mathrm{Kg} / \mathrm{m}^{2}$, e 30 a $35 \mathrm{Kg} /$ $\mathrm{m}^{2}$, respectivamente) e alguns ainda terão índices de obesidade mórbida, a depender da gravidade da doença à época da cirurgia17-23.

Vários autores associam em suas casuísticas índices maiores de complicações, sobretudo para torsoplastias ou abdominoplastias, no grupo de pacientes que ainda sofre de obesidade ${ }^{24-29}$. As complicações incluem seroma, hematoma, infecção, necrose gordurosa, necrose marginal da pele, deiscências de pele, necessidade de transfusão sanguínea e tempo prolongado de internação hospitalar. É interessante observar que as taxas de complicação afetam cerca de 35\% do grupo não-obeso (IMC de normal a sobrepeso) e até $80 \%$ dos que mantêm algum grau de obesidade.

As complicações sistêmicas, potencialmente mais severas, como a trombose venosa profunda e a embolia pulmonar, também assumem proporções superiores no grupo que, mesmo após sucesso terapêutico da cirurgia bariátrica, ainda apresenta obesidade. Índices reportados para trombose venosa profunda de $0,04 \%$ a $2,9 \%$ na população geral submetida a abdominoplastias podem atingir 8,9 \% no segmento pós-perda ponderal que ainda mantém obesidade. A necessidade de medidas preventivas é uma constante entre os autores, que recomendam os dispositivos de compressão intermitente da panturrilha no transoperatório, uso de heparina profilática, meias de compressão no pós-operatório, além de deambulação precoce e redução do tempo cirúrgico ${ }^{29-33}$.

A redução do tempo cirúrgico exige grande sincronização das equipes médicas. Em hospitais de missão universitária, isso permite realizar procedimentos combinados, tais como pexias mamárias e abdominoplastias, em tempos reduzidos, pelo uso de times simultâneos compostos de até quatro cirurgiões ${ }^{34}$. No paciente após perda ponderal maciça, pelas peculiaridades locais e sistêmicas, a padronização e treinamento são bons instrumentos para minimizar os riscos, além da criteriosa seleção de candidatos a procedimentos mais extensos. 


\section{Figura 1- Paciente de 31 anos, sexo feminino, IMC pré-gastroplastia de $56,1 \mathrm{~kg} / \mathrm{m}^{2}$ e IMC antes da cirurgia plástica de $29,2 \mathrm{~kg} / \mathrm{m}^{2}$. A e B: pré e pós-operatório de abdominoplastia. \\ C e D: pré e pós-operatório de dermolipectomia de face medial de coxas}

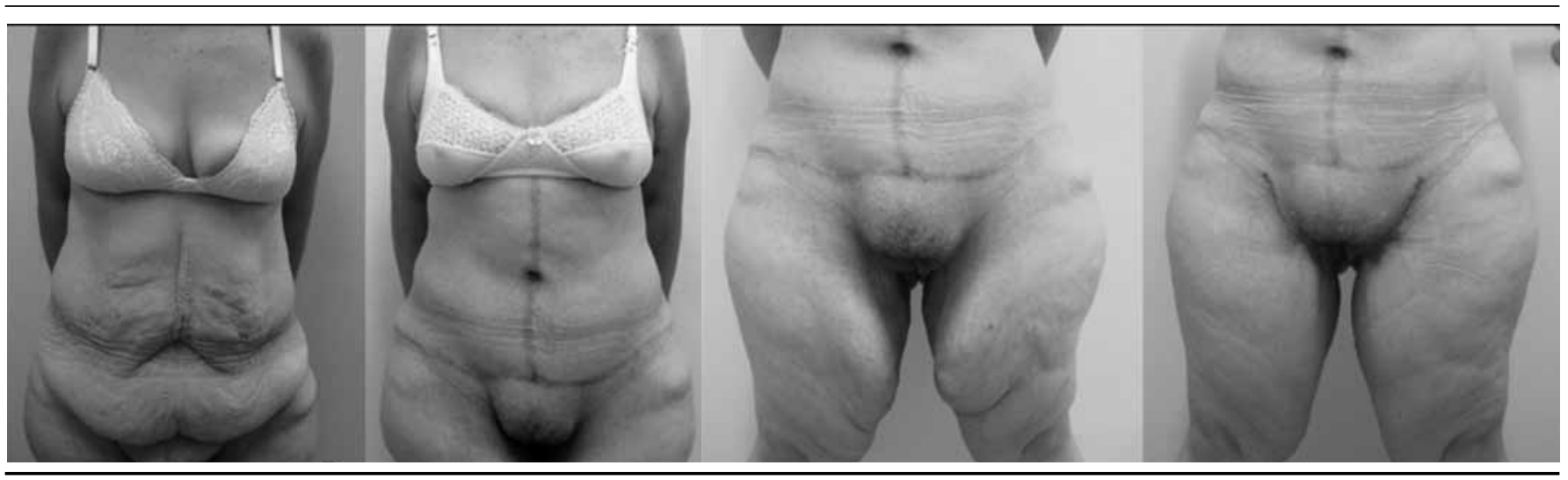

Figura 2- Paciente de 35 anos, sexo masculino, IMC pré-gastroplastia de $78,4 \mathrm{~kg} / \mathrm{m}^{2}$ e IMC antes da cirurgia plástica de $32,8 \mathrm{~kg} / \mathrm{m}^{2}$. A e B: pré-operatório. C e D: pós operatório de abdominoplastia

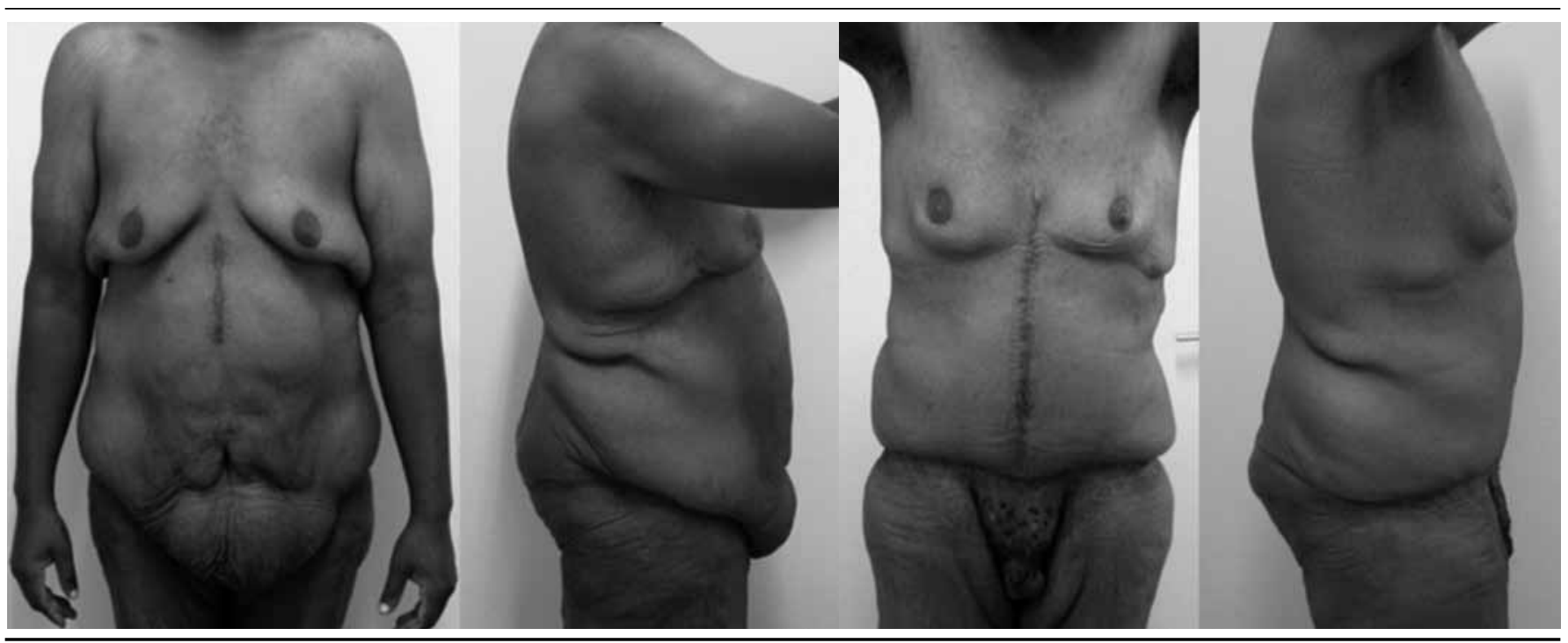

Há estudos em população geral submetida à abdominoplastia que, diferentemente das suposições gerais, não relacionam o tempo de prática profissional do cirurgião com seus índices de complicação ${ }^{28}$. Esse tipo de estudo ainda não foi desenvolvido para os pacientes após perda ponderal maciça.

As complicações pós-operatórias locais cutâneas estão entre as mais frequentes em candidatos às diversas técnicas de abdominoplastia e torsoplastia ${ }^{35-40}$. O conhecimento das regiões de vascularização da parede abdominal, a presença de cicatrizes prévias de laparotomias para hernioplastias ou colecistectomias, o cuidado técnico para evitar descolamentos excessivos e o nãotabagismo são fundamentais para uma cicatrização adequada.

O seroma é outra condição encontrada no pós-operatório, embora sua frequência varie entre os autores. Preconiza-se desde o uso de compressão local pós-operatória aos recentemente criados dispositivos de fluxo de gás ionizado de alta energia com intuito de interromper o extravazamento capilar de sangue e linfa41${ }^{44}$. Entretanto, ainda não há prevenção ideal além do cuidado na manipulação tecidual para evitar dissecação e lipólise excessivas.

Com o aumento da procura por cirurgias plásticas no grupo de pacientes após perda ponderal maciça, cresceu também a preocupação dos autores em identificar outros fatores de risco que possam comprometer resultados. Têm sido descritos maiores índices de complicação no sexo masculino e sobretudo em cirurgias múltiplas combinadas, quando mais de três regiões corporais são tratadas simultaneamente ${ }^{45,46,47}$.

Embora muito se discuta sobre o caráter estético ou reparador do tratamento destes pacientes e como as companhias de 

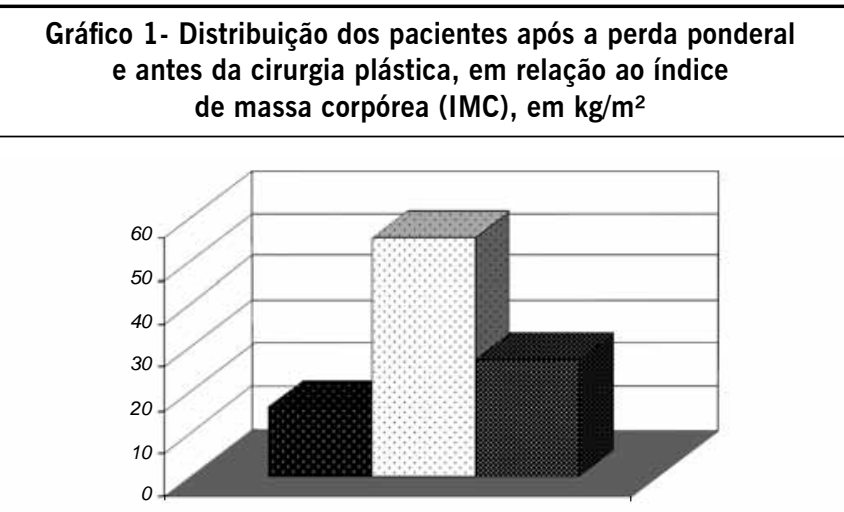

\begin{tabular}{|ll|}
\hline$I M C<25$ & 16 \\
\hline$\square I M C 25-30$ & 55 \\
\hline$I M C>30$ & 27 \\
\hline
\end{tabular}

seguro saúde se comportam em relação à realização das cirurgias plásticas $^{48-51}$, a busca por melhores resultados estéticos nesse âmbito perfaz outra constante preocupação dos autores. Novas técnicas para minimizar as cicatrizes, associação de técnicas de lipoaspiração, entre outras, são armamentos na conquista de linhas corporais mais harmoniosas para o paciente após emagrecimento maciço ${ }^{52-57}$.

A seleção criteriosa dos candidatos à cirurgia de contorno corporal é fundamental, sobretudo em relação às expectativas estéticas viáveis. Diversas estudos relatam maior frequência de psico-patologias neste grupo de pacientes, incluindo depressão, fobia social, ansiedade generalizada, desordem obsessivacompulsiva, justificando a importância da abordagem multidiscipinar com equipe de psicólogos ${ }^{58,59}$. Contudo, também se relata melhora na qualidade de vida e auto-imagem após o tratamento que inclui as cirurgias plásticas ${ }^{60}$.

Um grande empecilho aos resultados ideais neste grupo de pacientes é a constatação clínica da má qualidade cutânea, que após alguns meses de pós-operatório exibe flacidez residual desconcertante para o paciente e o cirurgião ${ }^{35}$. A obesidade é responsável por modificações na função de barreira da pele, nas excreções sebáceas e sudoríparas, na cicatrização das feridas, na microcirculação, e também na deposição protéica na derme, incluindo colágeno elastina ${ }^{61-66}$.

Alguns estudos em cadáveres analisaram a constituição da pele em relação à quantidade e qualidade de seu componente protéico (colágeno e elastina), com o objetivo de encontrar respostas para futuras comparações entre as diferentes capacidades de retração após cirurgias de lipoaspiração, por exemplo, no abdome e na região lateral de coxa ${ }^{67,68}$. Não há estudos dessa natureza em relação ao paciente após perda ponderal maciça, e para avaliar a constituição proteica da derme desses pacientes o autor está desenvolvendo pesquisa na área como projeto de pósgraduação em nível de mestrado a ser apresentado à Faculdade de Medicina da Universidade de São Paulo.

\section{Conclusão}

A obesidade figura atualmente como alarmante morbidade nas nações ocidentais. Os custos do tratamento da própria doença e das patologias a ela associadas são elevados e requerem investimentos amplos dentro do sistema de saúde. O advento da cirurgia bariátrica trouxe resultados duradouros e satisfatórios no combate a essa doença. O sucesso no tratamento da obesidade mórbida gera uma distorção na imagem corporal representada por excesso e flacidez cutâneos generalizados. O desejo de realizar cirurgias plásticas de contorno corporal para melhora desta situação atinge o paciente após perda ponderal maciça, brevemente no curso do seu tratamento. Cabe ao cirurgião plástico preparar-se para atender a essa demanda crescente, conhecendo as peculiaridades clínicas e os riscos maiores de complicações desse paciente, ponderar suas expectativas, analisar resultados e manter a busca constante pelo aperfeiçoamento das técnicas cirúrgicas.

\section{Conflito de interesse: não há}

\section{SUMMARY}

BODY CONTOUR SURGERY IN THE MASSIVE WEIGHT LOSS PATIENT: THREE YEAR-EXPERIENCE IN A SECONDARY PUBLIC HOSPITAL

OBJECTIVE. To present the clinical-surgical three year-experience related to body contour surgery in the massive weight loss patient, performed by the Plastic Surgery team of the Sapopemba State Hospital.

METHODS. Retrospective study performed at the Sapopemba State Hospital, linked to the "Hospital das Clínicas" of the University of São Paulo School of Medicine, from July 2005 to July 2008. A total of 98 patients was operated, whose morbid obesity had been treated by bariatric surgery, after success of the technique, or weight loss by non-surgical method. Exclusion criteria were smoking, gestational intent and weight instability. Stable weight for at least 6 months after satisfactory weight loss was required from patients.

RESULTS. Of the 98 operated patients, $97 \%$ were female, with mean age of 40.5 years and the weight loss method was the surgery in $88 \%$ of cases. From 177 plastic surgeries performed, $46 \%$ were abdominoplasty, followed by mammaplasty (15\%), thigh suspension (13\%) and brachioplasty (12\%). Mean hospital stay was of two days and the main diagnosed complications were: seroma (28\%), minor necrosis/dehiscences (18\%), major necrosis/dehiscences (4\%), hematomas (4\%), superficial thrombophlebitis from the saphena vein (2\%) and deep venous thrombosis (1\%).

CONCLUSION. An increasing demand for surgeries to improve body contour after massive weight loss has been detected. Plastic surgeons must know the clinical peculiarities and higher risk of complications of these patients, control their expectations, analyze results and engage in a continued investigation to improve surgical techniques. [Rev Assoc Med Bras 2009; 55(4): 427-33]

KEY WORDS: Plastic Surgery. Morbid obesity. 


\section{REFERÊNCIAS}

1. Buchwald H, Williams SE. Bariatric surgery worldwide 2003. Obes Surg. 2004;14:1157-64.

2. Davis TS. Morbid obesity. Clin Plast Surg. 1984;11:517-24.

3. Hamad GG. The state of art in bariatric surgery for weight loss in the morbidly obese patient. Clin Plast Surg. 2004;31:591-600.

4. Kreider JW, Hughes KC, Smeal D, Hirai T, Manders EK. Obesity Clin Plast Surg. 1996;23:671-81.

5. Pender JR, Pories WJ. Epidemiology of obesity in the United States. Gastroenterol Clin North Am. 2005;34:1-7.

6. Salameh JR. Bariatric surgery: past and present. Am J Med Sci. 2006;33(4):194-200.

7. Santry HP, Gillen DL, Lauderdale DS. Trends in bariatric surgical procedures. JAMA. 2005;294:1909-17.

8. Gmur RU, Banic A, Erni D. Is it safe to combine abdominoplasty with other dermolipectomy procedures to correct skin excess after weight loss? Ann Plast Surg. 2003;51:353-7.

9. Junqueira LC, Carneiro J. Histologia básica. $7^{a}$ ed. Rio de Janeiro: Guanabara Koogan; 1990. p.65-9, 271-6.

10. Mathes SJ. Plastic surgery. Philadelphia; 2006. p.294-6.

11. Alberts B, Bray D, Lewis J, Raff M, Roberts K, Watson JD. The cell. 3rd ed. New York, (NY): Garland Science; 1994. p.978-86.

12. Stryer L. Bioquímica. 3a ed. Rio de Janeiro: Guanabara-Koogan; 1992. p. 213-24.

13. Montes GS. Structural biology of the fibres of the collagenous and elastic systems. Cell Biol Int. 1996;20:15-27.

14. Ushiki T. Collagen fibers, reticular fibers and elastic fibers. A comprehensive understanding from a morphological viewpoint. Arch Histol Cytol. 2002;65:109-26.

15. Meigel WN, Gay S, Weber L. Dermal architecture and collagen type distribution. Arch Dermatol Res. 1977;259:1-10.

16. Tajima S, Nishikawa T, Hatano H, Nagai Y. Distribution of macromolecular components in human dermal connective tissue. Arch Dermatol Res. 1982;273:115-20

17.Shermak MA. Hernia repair and abdominoplasty in gastric bypass patients. Plast Reconstr Surg. 2006;117:1145-50.

18. Shons AR. Plastic reconstruction after bypass surgery and massive weight loss. Surg Clin North Am. 1979;59:1139-52.

19. Biaunie G, Kalis B. Cutaneous complications of massive obesity. Rev Prat. 1993;43:1930-4.

20. Hurwitz DJ, Agha-Mohammadi S. Postbariatric surgery breast reshaping: the spiral flap. Ann Plast Surg. 2006;56(5):481-6; discussion 486

21. Song AY, Jean RD, Hurwitz DJ, Fernstrom MH, Scott JA, Rubin JP. A classification of contour deformities after bariatric weight loss: the Pittsburgh rating scale. Plast Reconstr Surg. 2005;116:1535-44; discussion 1545-6.

22. Rubin JP, Nguyen V, Schwentker A. Perioperative management of the post gastric bypass patient presenting for body contour surgery. Clin Plast Surg. 2004;31:601-10

23. Sanger C, David LR. Impact of significant weight loss on outcome of body contouring surgery. Ann Plast Surg. 2006;56:9-13; discussion 13.

24. Manahan MA, Shermak MA. Massive panniculectomy after massive weight loss. Plast Reconstr Surg. 2006;117:2191-7, discussion 2198-9.

25. Rogliani M, Silvi E, Labardi L, Maggiulli F, Cervelli V. Obese and non obese patients complications of abdominoplasty. Ann Plast Surg. 2006;57:336-8.

26. Vastine VL, Morgan RF, Willians GS, Gampper TJ, Drake DB, Knox LK, Lin KY. Wound complications of abdominoplasty in obese patients. Ann Plast Surg. 1999;42:34-9.

27. Matarasso A, Swift RW, Rankin M. Abdominoplasty and abdominal contour surgery: a national plastic surgery survey. Plast Reconstr Surg. 2006;117(6): 1797-808.

28. Hunstad JP. Body contouring in the obese patient. Clin Plast Surg. 1996;23:647-70.

29. Aly AS, Cram AE, Heddens C. Truncal body contouring surgery in the massive weight loss patient. Clin Plast Surg. 2004;31(4):611-24.

30. Menderes A, Baytekin C, Haciyanli M, Yilmaz M. Dermalipectomy for body contouring after bariatric surgery in Aegean region of Turkey. Obes Surg. 2003;13:637-41.

31. Carwell GR, Horton CE. Circumferential torsoplasty. Ann Plast Surg. 1997;38(3):213-6.

32. Shermak MA, Chang DC, Heller J. Factors impacting thromboembolism after bariatric body contouring surgery. Plast Reconstr Surg. 2007;119:1590-96.
33. Rhomberg M, Pulzl P, Piza-Katzer H. Single stage abdominoplasty and mastopexy after weight loss following gastric banding. Obes Surg. 2003;13:418-23.

34. Modolin M, Cintra W Jr, Gobbi Cl, Ferreira MC. Circunferential abdominoplasty for sequential treatment after morbid obesity. Obes Surg. 2003;13:95-100.

35. Schechner SA, Jacobs JS, O'Loughlin KC. Plastic and reconstructive body contouring in post vertical banded gastroplasty patients: a retrospective review. Obes Surg. 1991;1:413-7.

36. Sagrillo D, Kunz S. Addressing skin redundancy after major weight loss. Plast Surg Nurs. 2004;24:130-3.

37. Costa LF, Landecker A, Manta AM. Optimizing body contour in massive weight loss patients: the modified vertical abdominoplasty. Plast Reconstr Surg. 2004;114:1917-23; discussion 1924-6.

38. Soundararajan V, Hart NB, Royston CM. Abdominoplasty following vertical banded gastroplasty for morbid obesity. Br J Plast Surg. 1995;48:423-7.

39. Savage RC. Abdominoplasty following gastrointestinal bypass surgery. Plast Reconstr Surg. 1983;71:500-9.

40. Pitanguy I, Gontijo de Amorim NF, Radwanski HN. Contour surgery in the patient with great weight loss. Aesthetic Plast Surg. 2000;24:406-11.

41. Ianelli A, Bafghi AR, Patrono D, Sautot-Vial N, Gugenheim J. Use of PlasmaJet system in patients undergoing abdominal lipectomy following massive weight loss resulting from bariatric surgery: early experience. Obes Surg. 2006;16:1504-7.

42. Vandeweyer E, Van Geertruyden J, De Fontaine S, Duchateau J, Houben JJ, Goldschmidt D. Post gastroplasty plastic surgery. Rev Med Brux. 1996;17:244-7.

43. Nemerofsky RB, Oliak DA, Capella JF. Body lift: an account of 200 consecutive cases in the massive weight loss patient. Plast Reconstr Surg. 2006;117:414-30.

44. Shermak MA, Chang D, Magnuson TH, Schweitzer MA. An outcomes analysis of patients undergoing body contouring surgery after massive weight loss. Plast Reconstr Surg. 2006;118:1026-31.

45. Strauch B, Herman C, Rohde C, Baum T. Mid body contouring in the post bariatric surgery patient. Plast Reconstr Surg. 2006;117:2200-11.

46. Hurwitz DJ. Single staged total body lift after massive weight loss. Ann Plast Surg. 2004;52:435-41; discussion 441.

47. Knoetgen $\mathrm{J} 3^{\text {rd }}$, Petty $P M$, Johnson $\mathrm{CH}$. Plastic surgery after bariatric surgery and massive weight loss. Mayo Clin Proc. 2005;80:136; author reply 136 .

48. Scheufler O, Erdmann D. Plastische chirurgie nach forcierter gewichtsreduktion- kosmetik oder rekonstruktion. Chirurg. 2007;78:316-25.

49. Heitmann C, Germann G. Plastisch-chirurgische korrekturen der korperformrekonstruktion und asthetik. Chirurg. 2007;78:273-86.

50. Rohrich RJ, Gosman AA, Conrad MH, Coleman J. Simplifying circumferential body contouring: the central body lift evolution. Plast Reconstr Surg. 2006;118:525-35.

51. Hurwitz DJ, Rubin JP, Risin M, Sajjadian A, Sereika S. Correcting the saddlebag deformity in the massive weight loss patient. Plast Reconstr Surg. 2004;114:1313-25.

52. Hurwitz DJ, Holland SW. The L-brachioplasty: an innovative approach to correct excess tissue of the upper arm, axilla, and lateral chest. Plast Reconstr Surg. 2006;117:403-11.

53. Rosik $\mathrm{CH}$. Psychiatric symptoms among prospective bariatric surgery patients: rates of prevalence and their relation to social desirability, pursuit of surgery, and follow up attendance. Obes Surg. 2005;15:677-83.

54. Kinzl JF, Traweger C, Trefalt E, Biebl W. Psychosocial consequences of weight loss following gastric banding for morbid obesity. Obes Surg. 2003;13:105-10.

55. Costa MP, Ching AW, Ferreira MC. Thin superior medial pedicle reduction mammaplasty for severe mammary hypertrophy. Aesthetic Plast Surg. 2008;32:645-52.

56. Costa MP, Tuma P Jr, Ferreira MC. Reduction mammaplasty with resection of a trapezoid base prism and adduction of the arms. Plast Reconstr Surg. 2005; 116:242-50.

57. Ferreira MC, Costa MP, Cunha MS, Sakae E, Fels KW. Sensibility of the breast after reduction mammaplasty. Ann Plast Surg. 2003;51:1-5.

58. Song AY, Rubin JP, Thomas V, Dudas JR, Marra KG, Fernstrom MH. Body image and quality of life in post massive weight loss body contouring patients. Obesity. 2006;14(9):1626-36.

59. Malheiros CA, Rodrigues FCM. Quando indicar cirurgia para obesidade mórbida? Rev Assoc Med Bras. 2000;46:303.

60. Cintra W Jr, Modolin ML, Gemperli R, Gobbi Cl, Faintuch J, Ferreira MC. Quality of life after abdominoplasty in women after bariatric surgery. Obes Surg. 2008;18:728-32. 
61. Yosipovitch G, DeVore A, Dawn A. Obesity and the skin: skin physiology and skin manifestations of obesity. J Am Acad Dermatol. 2007;56:901-20.

62. Sakuraoka K, Tajima S, Seyama Y, Teramoto K, Ishibashi M. Analysis of connective tissue macromolecular componentes in ishibashi rat skin: role of collagen and elastin in cutaneous aging. J Dermatol Sci. 1996;12:232-7.

63. Dalferes ER Jr, Radhakrishnamurthy B, Cruch MS, Berenson GS. A study of connective tissue macromolecules in skin of mice with goldthioglucose induced obesity. Proc Soc Exp Biol Med. 1975;148:918-24.

64. Enser N, Avery NC. Mechanical and chemical properties of the skin and its collagen from lean and obese-hyperglycaemic mice. Diabetologia. 1984;27:44-9.

65. Rasmussen MH, Jensen LT, Andersen T, Breum L, Hilsted J. Collagen metabolism in obesity: the effect of weight loss. Int J Obes Relat Metab Disord. 1995;19:659-63.
66. Modolin ML, Cintra W Jr, Paggiaro AO, Faintuch J, Gemperli R, Ferreira MC. Massive localized lymphedema (MLL) in bariatric candidates. Obes Surg. 2006; $16: 1126-30$

67. Goodson MH III, Hunt TK. Wound collagen accumulation in obese hyperglycemic mice. Diabetes. 1986:35:491-5.

68. Faria JC, Tuma Jr P, Costa MP, Quagliano AP, Ferreira MC. Skin aging and collagen. Rev Hosp Clin Fac Med São Paulo. 1995;50(suppl):39-43.

Artigo recebido: 03/08/08

Aceito para publicação: 04/02/09 